\title{
Approximating the Stability Region for a Differential Equation with a Distributed Delay
}

\author{
S.A. Campbell* and R. Jessop \\ Department of Applied Mathematics, University of Waterloo, Waterloo, N2L 3G1, Canada
}

\begin{abstract}
We discuss how distributed delays arise in biological models and review the literature on such models. We indicate why it is important to keep the distributions in a model as general as possible. We then demonstrate, through the analysis of a particular example, what kind of information can be gained with only minimal information about the exact distribution of delays. In particular we show that a distribution independent stability region may be obtained in a similar way that delay independent results are obtained for systems with discrete delays. Further, we show how approximations to the boundary of the stability region of an equilibrium point may be obtained with knowledge of one, two or three moments of the distribution. We compare the approximations with the true boundary for the case of uniform and gamma distributions and show that the approximations improve as more moments are used.
\end{abstract}

Key words: delay differential equations, distributed delay, linear stability, delay independent stability

AMS subject classification: 34K20, 34K06, 92B05, 92B20

\section{Introduction}

Time delays are an important aspect of many biological and physical models. In models for population growth, delays typically arise due to age structure, e.g. due to the time spent in a larval stage before becoming an adult or due to the gestation period [14]. In epidemic models, delays arise as

\footnotetext{
${ }^{*}$ Corresponding author. E-mail: sacampbell@uwaterloo.ca
} 
a result of the time spent in each stage of the disease, e.g. when someone becomes infected with a disease they do not recover instantaneously but only after some period of time $[8,3]$. In models for neural systems, there is a delay (the propagation delay) due to the time it takes for the signal (action potential) of one neuron to travel from the soma of the sending neuron to synapse and from the synapse to the soma of the receiving neuron. There is also a delay (the processing delay) due to the time it takes for the signal to cross a chemical synapse between the neurons [21, 27].

Many models, especially those for neural systems, assume that the time delays are fixed. For example, Hutchinson [19] proposed the following modification of the logistic equation

$$
\dot{x}(t)=r x(t)\left[1-\frac{x(t-\tau)}{K}\right],
$$

as a model for a population of insects. Here the delay $\tau$ represents the time between egg laying and hatching. We shall refer to such models as discrete delay models. It is more likely, however, that the delay will vary with some individuals taking more time to hatch and some taking less. Thus a more appropriate model includes a distribution of delays, representing the situation where the delay occurs in some range of values with some associated probability distribution, viz.,

$$
\dot{x}(t)=r x(t)\left[1-\frac{1}{K} \int_{0}^{\infty} x(t-u) g(u) d u\right] .
$$

The function $g$ in this model is called the kernel of the distribution and represents the probability that a particular time delay occurs. Since $g$ is a probability density function, it is normalized so that $\int_{0}^{\infty} g(u) d u=1$. Note that if $g$ is taken to be a Dirac distribution, i.e., $g(u)=\delta(u-\tau)$, we recover the model with discrete delay.

In the literature, there are two commonly used distributions. The uniform distribution with mean $\tau$ is given by

$$
g(u)=\left\{\begin{array}{cc}
\frac{1}{\tau \rho}, & \text { for } \tau\left(1-\frac{\rho}{2}\right) \leq u \leq \tau\left(1+\frac{\rho}{2}\right) \\
0, & \text { elsewhere }
\end{array}\right.
$$

The parameter $\rho$ determines the width and height of the distribution. Some examples of this distribution are shown in Figure 1(a). The gamma distribution is given by

$$
g(u)=\frac{u^{p-1} a^{p} e^{-a u}}{\Gamma(p)},
$$

where $a, p \geq 0$ are parameters determining the shape of the distribution. In particular, the mean is given by $\tau=p / a$. $\Gamma$ is the gamma function defined by $\Gamma(0)=1$ and $\Gamma(p+1)=p \Gamma(p)$. Some examples of this distribution are shown in Figure 1(b). It is common to consider $p$ in the gamma distribution to be an integer. In this case $\Gamma(p)=(p-1)$ ! and a scalar equation with one gamma distributed delay can be shown to be equivalent to a system of $p+1$ ordinary differential equations. Details about this reduction, often called the linear chain trick, may be found in the books of MacDonald [24, 25]. This approach may seem attractive as it means the model is amenable to analysis and simulation techniques for ordinary differential equations. However, it will only be practical if $p$ is a small integer, while real data may call for much larger and/or noninteger $p$. As 


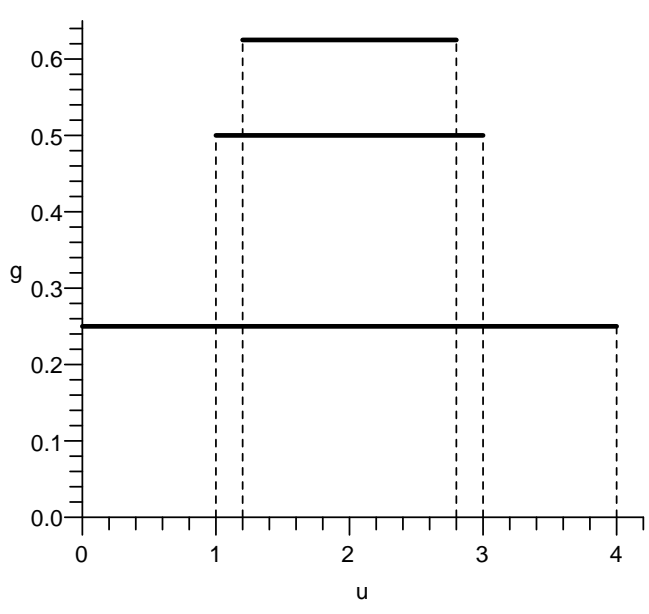

(a) Uniform distribution

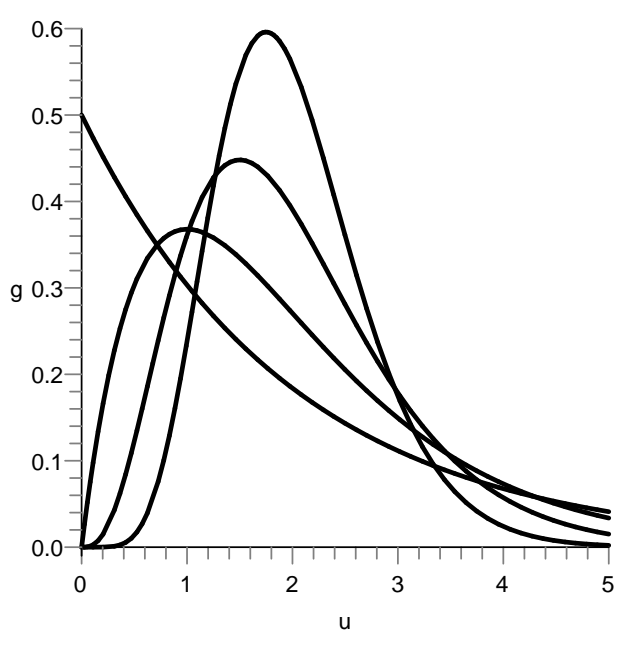

(b) Gamma distribution

Figure 1: (a) The uniform distribution for $\rho=0.8,1,2$ and mean delay $\tau=2$. (b) The gamma distribution for $p=1,2,4,8$ and mean delay $\tau=\frac{p}{a}=2$.

an example we note a recent paper [33] where data for the pre-symptomatic infectious period in an outbreak of mumps was fit by a gamma distribution with $p=70$.

Models with distributed delays have been considered in the population biology and epidemic literature for some time, but only recently in the neural network literature. In the majority of this literature (e.g. [23, 29]) specific kernels (usually the gamma distribution with $p=1$ or $p=2$ ) are put into the model and the resulting behaviour is analyzed in detail. Such papers are useful in adding to our general knowledge of how distributed delays affect the dynamics differently from discrete delays. However, if one is interested in modelling real physical systems, one usually does not have access to the exact distribution, and approaches using general kernels may be more useful. This is the point of view we will take in this article and thus we begin with a review of the literature where results for distributed delay equations with general kernels have been obtained.

There are several monographs which explicitly treat systems with distributed delays. The monograph of Cushing [14] gives a general introduction to the study of systems with a distribution of delays, as well as stability and bifurcation analysis of some specific biological models. Some linear stability results for general distributions are derived. The monographs of MacDonald $[24,25]$ give linear stability results for system with gamma distributions (for general $p$ ). The monograph of Gopalsamy [16] uses the Liapunov function approach to derive some stability results for models from population biology. The monograph of Kuang [22] also treats equations with distributed delay.

In the population biology literature there are several papers which give results for general distributions. Faria and Oliveira [15] study the global stability of equilibria in a class of Lotka-Volterra models with distributed delays having finite maximum delay. They give conditions on the inter- 
action coefficients of the system which guarantee asymptotic stability for any distribution. Global stability analysis of the equilibria in a chemostat model with gamma distributed delay has been carried out by Wolkowicz et al. [31,32]. They obtain results for any gamma distribution and show that if in the limit the variance goes to zero with the mean delay held fixed, the results yield those for the system with discrete delay. This fact was observed for linear stability by MacDonald [25]. They also note that changing the mean delay has a much larger effect on stability than changing the variance. Ruan [28] considers Hutchinson's equation with distributed delay, i.e., equation (1.1) and gives a condition for asymptotic stability of the trivial solution in terms of the mean of the distribution. Arino and van den Driessche [3] show how the reproduction number for certain epidemic models with distributed delays can be derived without assuming a particular form for the distribution.

In the neural network literature, there are fewer results. Gopalsamy and He [17] study global stability of equilibria in a Hopfield-type network of arbitrary size with either discrete time delays or gamma distributed time delays. Chen $[10,11]$ studies global stability of equilibria in artificial neural networks with distributed delays with arbitrary kernels using Liapunov functionals and matrix theory. Also, the work of Faria and Oliveira [15] discussed above can be viewed as the linearization of a Hopfield-type neural network about an equilibrium point. Thiel et al. [30] study a scalar equation representing a mean field approximation for a population of pyramidal cells with recurrent feedback, first formulated by [26]. They show that having a uniform distribution of delays simplifies the dynamics of the system. The size of the stability region of the equilibrium point is larger, and larger mean delays are needed to induce oscillations. Complex phenomena such as chaos are less likely to occur, or totally precluded if the variance of the distribution is sufficiently large. The model with a distribution of delays better explains the appearance of periodic bursts of activity when penicillin is added to a hippocampal slice preparation (which reduces the coupling strength).

An often quoted general principle is that a system with a distribution of delays is inherently more stable than the same system with a discrete delay. It is clear that some of the results cited above support this principle. Other are described below. Cooke and Grossman [13] and MacDonald [24] compare the behaviour of a scalar equation with one discrete delay:

$$
\dot{x}(t)=-a x(t)-b x(t-\tau)
$$

and the corresponding equation with a gamma distributed delay. They show that, for appropriate values of $a$ and $b$, increasing the discrete delay in the model destabilizes the trivial solution and it can never be restabilized. For the distributed delay, increasing the mean delay can also destabilize the trivial solution, however, it will always be restabilized for large enough mean delay, for any value of $p$. Jirsa and Ding [20] analyze an $n \times n$ linear system with linear decay and arbitrary connections with a common delay. They show, under some mild assumptions, that the stability region of the trivial solution for any distribution of delays is larger than and contains the stability region for a discrete delay. Campbell and Ncube [9] study a scalar equation with one discrete delay and one distributed delay:

$$
\dot{x}(t)=-k x(t)+\alpha x\left(t-\tau_{s}\right)+\beta \int_{0}^{\infty} x(t-\tau) g(\tau) d \tau .
$$


They show that it is more difficult to get delay induced instability with gamma distributions, than in the corresponding system with two discrete delays. For large variance $(p=1)$ delay induced instability is impossible, and for smaller variance $(p>1)$ the mean delay needed for instability is much larger than the discrete delay value. They also show that sufficiently small variance in the distribution is needed to get the stability switches observed in the corresponding system with two discrete delays. In [4, 5], Atay studies a system consisting of two simple oscillators with gap junctional coupling by incorporating a uniform distribution of delays. He shows it is easier to destroy oscillations with a distribution of delays than with a discrete delay, in the sense that there is a larger region of oscillator death in the parameter space consisting of the mean delay and the strength of the coupling. As the variance of the distribution increases, the size of this region increases. In [6] Atay studies the stability of functional differential equations near a Hopf bifurcation under delayed feedback. He shows the discrete delay is locally the most destabilizing one among delay distributions having the same mean. He also gives conditions under which the delays induce stability. In this case, he proves that the discrete delay is locally the most stabilizing.

Bernard et al. [7] have pointed out that it makes the most biological sense to incorporate a distribution that has a nonzero minimum delay, since the probability of having zero delay is effectively zero in most applications. For the uniform distribution, this can be achieved naturally by incorporating the constraint $\rho<2$. The gamma distribution can be reformulated to achieve this as follows:

$$
g(u)=\left\{\begin{array}{cc}
0, & \text { for } 0 \leq u<\tau^{\min } \\
\frac{a^{p}}{\Gamma(p)}\left(u-\tau^{\min }\right)^{p-1} e^{-a\left(u-\tau^{\min }\right)}, & \text { for } \tau^{\min } \leq u
\end{array}\right.
$$

where $\tau^{\min }>0$ is the minimum delay. In this case, the linear chain trick shows that one equation with a gamma distribution is equivalent to a system of $p$ ordinary differential equations and one discrete delay differential equation. Bernard et al. [7] analyze the linear stability of a scalar system with one and two delays in terms of generic properties of the distribution $g$, such as the mean, variance and skewness. For the uniform and continuous distributions, they have shown that stability regions are larger than those with a discrete delay. The equation studied by Bernard et al. [7] is similar to the linearization of the equation analyzed by Adimy et al. [1] describing a model of blood cell production in the bone marrow. Adimy et al. determine conditions that guarantee the local asymptotic stability of the nontrivial equilibrium and also obtain conditions for a Hopf bifurcation to occur for any general distribution of delays. Further, Adimy et al. [2] study a model of pluripotent stem cell population where the delay describing the cell cycle duration is distributed uniformly. They obtain stability conditions independent of delay and show that the distributed delay can destabilize the nontrivial equilibrium via a Hopf bifurcation. The linearization of their model is similar to the scalar equation we consider in this paper.

In the following we will show, through the analysis of a specific example, how one can obtain results for general distributions. The primary focus will be on the linear stability of equilibrium points of the equations, via analysis of the characteristic equation. These results are not only important for understanding where equilibrium points are stable, but also for determining where bifurcations of the equilibrium points occur.

The plan for the article is as follows. First we show what can be determined without choosing a particular distribution. Next we illustrate a way of approximating the region of stability when the 
actual distribution is not known, but some moments of the distribution are. Finally, we compare the approximate stability regions with the stability regions for the gamma and uniform distributions. For ease of presentation we will consider a simple scalar system, the extension to others system should be clear.

\section{Distribution independent results}

Consider the scalar DDE with one distributed delay

$$
\dot{x}(t)=\int_{0}^{\infty} f(x(t), x(t-u)) g(u) d u,
$$

where $\int_{0}^{\infty} g(u) d u=1$. Let the mean delay of the distribution be given by

$$
\tau=\int_{0}^{\infty} u g(u) d u .
$$

Biologically, the most interesting case is when $\tau>0$ and we will assume this from now on. The above equation includes many models. The distributed delay version of Hutchinson's equation given by equation (1.1) is one example. Assume that the system has an equilibrium point $x^{*}$, i.e.,

$$
0=\int_{0}^{\infty} f\left(x^{*}, x^{*}\right) g(u) d u=f\left(x^{*}, x^{*}\right) .
$$

Linearizing (2.1) about $x^{*}$ we obtain

$$
\dot{x}(t)=-\alpha x(t)-\beta \int_{0}^{\infty} x(t-u) g(u) d u,
$$

where $-\alpha$ is the derivative of $f$ with respect to its first argument, and $-\beta$ is the derivative of $f$ with respect to its second argument.

In the following, we would like to study the dependence of the linear stability of the equilibrium point on the mean delay. Thus we will transform the equation so that the mean delay appears explicitly. Let $s=t / \tau, v=u / \tau$ and ' denote the derivative with respect to $s$, then we have

$$
x^{\prime}(s)=-\tau \alpha x(s)-\tau \beta \int_{0}^{\infty} x(s-v) g(\tau v) \tau d v .
$$

Defining $\hat{g}(v)=g(\tau v) \tau$ we find

$$
x^{\prime}(s)=-\tau \alpha x(s)-\tau \beta \int_{0}^{\infty} x(s-v) \hat{g}(v) d v .
$$

Note that the equation is still in the form of a distributed delay, however the mean of the distribution $\hat{g}$ is 1 . 
Setting $x(s)=e^{\lambda s}$ we derive the characteristic equation

$$
\Delta(\lambda)=\lambda+\alpha \tau+\beta \tau \int_{0}^{\infty} e^{-\lambda v} \hat{g}(v) d v=0 .
$$

Note that this may be written

$$
\lambda+\alpha \tau+\beta \tau \hat{G}(\lambda)=0,
$$

where $\hat{G}$ is the Laplace transform of $\hat{g}$.

Changes of stability of the equilibrium point will take place when the characteristic equation has a root with zero real part. In the following we will describe where in the parameter space such changes may occur, and hence determine the region of stability of the equilibrium point. We will consider the parameter $\alpha$ as fixed and describe the stability region in the $\beta, \tau$ parameter space for various values of $\alpha$.

In the study of systems with discrete delays, it is common to describe the delay independent stability region, sometimes called the absolute stability region. Such results give a conservative estimate of the stability region which is useful if one is unable to accurately estimate the time delay in the system. In this section we will formulate similar results for equation (2.3). In particular we will give one result which is independent of all aspects of the distribution and one which is independent of all aspects save the mean delay. This latter may be especially useful as the mean delay is often all one may be able to estimate for a particular system. We begin with a result which will help locate the region of stability of the equilibrium point.

Theorem 1. Assume that $\hat{G}(\lambda)$ is analytic in $\operatorname{Re}(\lambda) \geq 0$. If $0<|\beta|<\alpha$ then the characteristic equation has no roots with positive real part. If $0<|\beta|<-\alpha$ then the characteristic equation has one root with positive real part.

Proof. We will prove the results by the use of Rouché's Theorem [12, p. 313]. Let $f_{1}(\lambda)=\lambda+\alpha \tau$ and $f_{2}(\lambda)=\beta \tau \hat{G}(\lambda)$, and consider the contour in the complex plane, $C=C_{1} \cup C_{2}$, given by

$$
\begin{gathered}
C_{1}: \lambda=R e^{i \theta},-\frac{\pi}{2} \leq \theta \leq \frac{\pi}{2} \\
C_{2}: \lambda=i y,-R \leq y \leq R
\end{gathered}
$$

where $R$ is a real number.

On $C_{1}$ we have $\left|f_{1}(\lambda)\right| \geq|R-| \alpha|\tau|$ and $\left|f_{2}(\lambda)\right| \leq|\beta| \tau$. Thus $\left|f_{1}(\lambda)\right|>\left|f_{2}(\lambda)\right|$ for $R$ sufficiently large. On $C_{2}$ we have $\left|f_{1}(\lambda)\right| \geq|\alpha| \tau$ and $\left|f_{2}(\lambda)\right| \leq|\beta| \tau$. Thus $\left|f_{1}(\lambda)\right|>\left|f_{2}(\lambda)\right|$ if $|\alpha|>|\beta|$. Further, note that if $\alpha \tau \neq 0$, and $\beta \tau \neq 0$ then both $f_{1}$ and $f_{2}$ do not reduce to zero anywhere on $C$. Thus by Rouché's Theorem, if $|\alpha|>|\beta|>0$ and $R$ is sufficiently large then $f_{1}(\lambda)$ and $\Delta(\lambda)=f_{1}(\lambda)+f_{2}(\lambda)$ have the same number of zeros inside $C$. Let $R \rightarrow \infty$ then $f_{1}(\lambda)$ and $\Delta(\lambda)$ have the same number of zeros with $\operatorname{Re}(\lambda)>0$. Now $f_{1}(\lambda)$ has just one zero at $\lambda=-\alpha \tau$. The results follow.

¿From this theorem, we can conclude that the trivial solution of equation (2.3) (and hence the equilibrium point of equation (2.1)) is locally asymptotically stable if $\alpha>0$ and $|\beta|<\alpha$ and 
unstable if $\alpha<0$ and $|\beta|<-\alpha$. We note that these results also hold for equation (2.2) and its discrete delay analogue

$$
\dot{x}(t)=-\alpha x(t)-\beta x(t-\tau) .
$$

We can next determine a region in the parameter space where the equilibrium point is unstable for any distribution $\hat{g}(v)$. The proof of the following result is similar to the proof of Theorem 3.1 in [1].

Theorem 2. The equilibrium point of (1) is unstable when $\beta<-\alpha$.

Proof. Note that we only need to consider the case when $\beta<-|\alpha|$, since from Theorem 1 we already have instability for $\alpha<\beta<-\alpha$, when $\alpha<0$. We focus on the real roots of the characteristic equation (2.4), hence we assume $\Delta(\lambda): \mathbb{R} \rightarrow \mathbb{R}$. Now

$$
\frac{d \operatorname{Re}(\Delta(\lambda))}{d \lambda}=1-\beta \tau \int_{0}^{\infty} v e^{-\lambda v} \hat{g}(v) d v>0
$$

since $\beta<0$. Thus $\Delta(\lambda)$ is a strictly increasing function. For $\lambda=0$ we have

$$
\Delta(0)=\tau(\alpha+\beta)<0,
$$

by our assumption. If $\lambda \geq 0$ then $\left|e^{-\lambda v}\right| \leq 1$, and thus the integral term is bounded:

$$
\int_{0}^{\infty} e^{-\lambda v} \hat{g}(v) d v \leq\left|\int_{0}^{\infty} e^{-\lambda v} \hat{g}(v) d v\right| \leq \int_{0}^{\infty}\left|e^{-\lambda v}\right| \hat{g}(v) d v \leq \int_{0}^{\infty} \hat{g}(v) d v=1 .
$$

It follows that

$$
\lim _{\lambda \rightarrow+\infty} \Delta(\lambda)=+\infty .
$$

Since $\Delta(\lambda)$ is continuous, we conclude that $\Delta(\lambda)$ has a unique real root which is positive, i.e. the characteristic equation has at least one root with positive real part. The result follows.

Some additional information about the stability region may be obtained from the characteristic equation (2.4). First note that the characteristic equation has a zero root if $\alpha+\beta=0$ for any distribution. For $\alpha>0$, from Theorems 1 and 2, stability is gained as this line is crossed by increasing the parameter $\beta$, and thus the line $\beta=-\alpha$ forms part of the boundary of the stability region. For $\alpha<0$ we need to determine how the eigenvalue changes as this line is crossed in the parameter space. We will focus on varying the parameter $\beta$, the analysis for the variation of $\alpha$ is similar.

To begin, we note that

$$
\frac{d \operatorname{Re}(\lambda)}{d \beta}=\operatorname{Re}\left(\frac{d \lambda}{d \beta}\right)=-\operatorname{Re}\left(\frac{\partial \Delta}{\partial \beta} / \frac{\partial \Delta}{\partial \lambda}\right) .
$$

In particular, we need to evaluate this when $\lambda=0$, i.e. along the line $\beta=-\alpha$ :

$$
\left.\frac{d \operatorname{Re}(\lambda)}{d \beta}\right|_{\lambda=0, \beta=-\alpha}=-\operatorname{Re}\left(\frac{\partial \Delta}{\partial \beta} /\left.\frac{\partial \Delta}{\partial \lambda}\right|_{\lambda=0, \beta=-\alpha}\right)=-\frac{\tau}{1+\alpha \tau} .
$$


¿From this it is easy to conclude that

$$
\frac{d \operatorname{Re}(\lambda)}{d \beta}>0 \text { along the line } \beta=-\alpha, \text { with } \alpha<0 \text { and } \tau>-\frac{1}{\alpha} .
$$

It follows that for $\alpha<0$ and $\tau<-1 / \alpha$, the equilibrium point becomes stable as $\beta$ is increased through the line $\beta=-\alpha$. Hence, for $\alpha<0$ the line segment $\beta=-\alpha, \tau<-1 / \alpha$ forms part of the stability boundary.

To further define the boundary of stability, we need to determine where the characteristic equation has a pair of pure imaginary roots, $\pm i \omega$. This occurs when we set $\lambda=i \omega, \omega>0$ in the characteristic equation (2.4), i.e.,

$$
i \omega+\alpha \tau+\beta \tau \int_{0}^{\infty} e^{-i \omega v} \hat{g}(v) d v=0 .
$$

Separating this into real and imaginary parts, we find that for the characteristic equation to have a pair of real imaginary roots, the parameters must satisfy the following equations

$$
\begin{aligned}
\alpha \tau & =-\beta \tau \int_{0}^{\infty} \cos (\omega v) \hat{g}(v) d v \stackrel{\text { def }}{=}-\beta \tau C(\omega), \\
\omega & =\beta \tau \int_{0}^{\infty} \sin (\omega v) \hat{g}(v) d v \stackrel{\text { def }}{=} \beta \tau S(\omega) .
\end{aligned}
$$

Fixing $\alpha$, we can formally define curves, parameterized by $\omega$, in the $\beta, \tau$ plane along which the equations in (2.8) are satisfied. These curves are given by

$$
\beta=-\frac{\alpha}{C(\omega)}, \quad \tau=-\frac{\omega}{\alpha} \frac{C(\omega)}{S(\omega)},
$$

for all $\omega>0$ such that $C(\omega)$ and $S(\omega)$ are nonzero. The values of $\omega$ such that $C(\omega)=0$ or $S(\omega)=0$ define discontinuities in the curves and do not correspond to roots of the characteristic equation (2.7).

To obtain explicit expressions for the curves given in (2.9), we need to evaluate $C(\omega)$ and $S(\omega)$ which requires knowledge of the distribution $\hat{g}(v)$. Without this knowledge, we can still determine how the number of eigenvalues changes as one crosses one of these curves. From equations (2.7) and (2.8), the characteristic equation when $\lambda=i \omega$ can be written as

$$
\Delta(i \omega)=i \omega+\alpha \tau+\beta \tau(C(\omega)-i S(\omega))=0 .
$$

Taking the derivative of $\tau$ in (2.9) with respect to $\omega$ we obtain

$$
\frac{d \tau}{d \omega}=-\frac{1}{\alpha S(\omega)}\left(C(\omega)+\omega \frac{C^{\prime}(\omega) S(\omega)-S^{\prime}(\omega) C(\omega)}{S(\omega)}\right) .
$$


Next we compute the rate of change of the real part of $\lambda$ with respect to $\beta$,

$$
\begin{aligned}
\left.\frac{d \operatorname{Re}(\lambda)}{d \beta}\right|_{\lambda=i \omega} & =-\operatorname{Re}\left(\frac{\partial \Delta}{\partial \beta} /\left.\frac{\partial \Delta}{\partial \lambda}\right|_{\lambda=i \omega}\right) \\
& =-\tau \operatorname{Re}\left(\frac{C(\omega)-i S(\omega)}{1-\beta \tau\left(S^{\prime}(\omega)+i C^{\prime}(\omega)\right)}\right) \\
& =-\frac{\tau}{H^{2}(\omega)}\left(C(\omega)-\beta \tau C(\omega) S^{\prime}(\omega)+\beta \tau S(\omega) C^{\prime}(\omega)\right) \\
& =-\frac{\tau}{H^{2}(\omega)}\left(C(\omega)+\omega \frac{C^{\prime}(\omega) S(\omega)-S^{\prime}(\omega) C(\omega)}{S(\omega)}\right),
\end{aligned}
$$

where $H^{2}(\omega)=\left(1-\beta \tau S^{\prime}(\omega)\right)^{2}+\left(\beta \tau C^{\prime}(\omega)\right)^{2}$ is a positive function of $\omega$ and we have used $\beta \tau=\omega / S(\omega)$ from (2.9). Comparing with (2.10), and using (2.9) again, we see that

$$
\left.\frac{d \operatorname{Re}(\lambda)}{d \beta}\right|_{\lambda=i \omega}=\frac{\alpha}{\beta} \frac{\omega}{H^{2}(\omega)} \frac{d \tau}{d \omega} .
$$

Thus whether the number of eigenvalues with positive real part is increasing or decreasing as $\beta$ is increased through a point on one of the curves defined by (2.9) depends on the sign of $\alpha$ and whether $\tau$ is an increasing or decreasing function of $\omega$ at the point.

We can also obtain the following distribution independent results.

Theorem 3. Under the conditions of Theorem 1, the equilibrium point of (1) is locally asymptotically stable in the following regions of parameter space

$$
\begin{aligned}
& \text { (1) }|\beta|<\alpha \\
& \text { (2) }|\alpha| \leq \beta \text { and } 0<\tau<\frac{1}{\beta} .
\end{aligned}
$$

Proof. Result (1) follows from Theorem 1 and the subsequent discussion. To see (2), consider equations (2.8). ¿From the first equation, we have

$$
\left|\frac{\alpha}{\beta}\right| \leq \int_{0}^{\infty}|\cos (\omega v)| \hat{g}(v) d v \leq \int_{0}^{\infty} \hat{g}(v) d v=1
$$

While from the second equation, we have

$$
\left|\frac{1}{\beta \tau}\right| \leq \int_{0}^{\infty}\left|\frac{\sin (\omega v)}{\omega v}\right| v \hat{g}(v) d v \leq \int_{0}^{\infty} v \hat{g}(v) d v=1 .
$$

Thus equations (2.8) have a solution only if $|\beta| \geq|\alpha|$ and $\tau \geq 1 /|\beta|$. In particular, for $\beta>0$ this means the system cannot have pure imaginary roots if $\beta \geq|\alpha|$ and $\tau<1 / \beta$ and hence for this range of $\beta$, the stability cannot change. Result (2) follows.

We note that, in the case $\alpha>0$, the second result of this theorem is similar to Theorem 9 in [28], although we have proven it in a different way. 


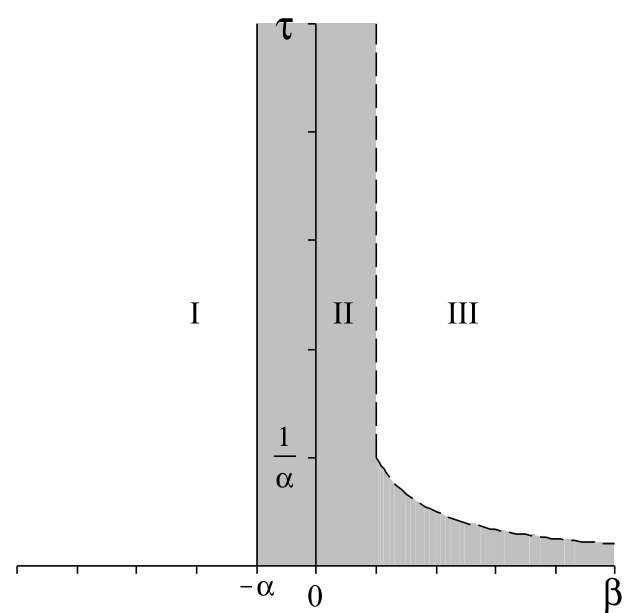

(a) $\alpha>0$

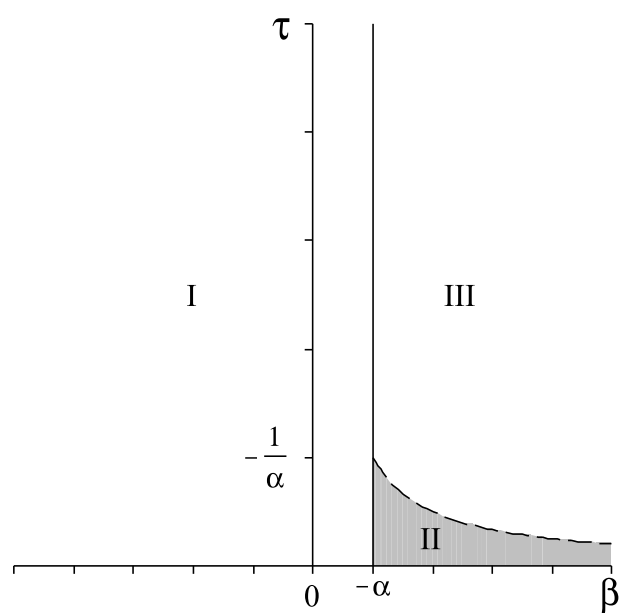

(b) $\alpha<0$

Figure 2: Illustration of the distribution independent stability results described by Theorems 2 and 3. (I) Region of instability described by Theorem 2. (II) Region of stability described by Theorem 3. (III) No distribution independent stability results are known for this region.

The results of Theorems 2 and 3 are illustrated in Figure 2. Theorem 3 describes the region of stability of the equilibrium point with either no knowledge of the distribution of delays or knowledge of only the first moment of the distribution, i.e. the mean delay, $\tau$. ¿From the proof of Theorem 3, it is clear that the curves given by equations (2.9) must lie outside the stability region described by the theorem. Thus this region is only a conservative estimate of the full region in the parameter space where the equilibrium point is stable. In the next section we will show how one may improve this approximation by using more information from the distribution. We note that it is only necessary to consider $\beta>0$, given the results of Theorems 2 and 3 .

\section{Approximating the boundary of the stability region}

In the previous section, we established that the boundary of the stability region (in the $\beta, \tau$ plane) consists of all or part of the line $\beta=-\alpha$ and the curve(s) defined parametrically by equations (2.9) for $\beta>|\alpha|$. In this section, we shall show how partial knowledge of the distribution, $\hat{g}(v)$, can allow us to approximate these latter curves.

Let $m_{j}$ denote the $j^{\text {th }}$ moment of $\hat{g}$, i.e.,

$$
m_{j}=\int_{0}^{\infty} v^{j} \hat{g}(v) d v
$$

It is easy to check that $m_{0}=m_{1}=1$ and that $m_{j}>0$ for all $j$. The second moment is related to the variance, $\sigma^{2}$, via $m_{2}=\sigma^{2}+1$. And the third moment is related to the measure of the 
Table 1: Approximations for equations (2.9), where $M$ and $N$ relate to the number of terms used in the summation for $C(\omega)$ and $S(\omega)$, respectively.

\begin{tabular}{|c||c|c|c|c|}
\hline$(M, N)$ & $C(\omega)$ & $S(\omega)$ & $\beta$ & $\tau$ \\
\hline$(0,0)$ & 1 & $\omega$ & $-\alpha$ & $-1 / \alpha$ \\
\hline$(1,0)$ & $1-\frac{m_{2}}{2} \omega^{2}$ & $\omega$ & $\frac{2 \alpha}{m_{2} \omega^{2}-2}$ & $\frac{m_{2} \omega^{2}-2}{2 \alpha}$ \\
\hline$(1,1)$ & $1-\frac{m_{2}}{2} \omega^{2}$ & $\omega-\frac{m_{3}}{6} \omega^{3}$ & $\frac{2 \alpha}{m_{2} \omega^{2}-2}$ & $\frac{3 m_{2} \omega^{2}-6}{6 \alpha-\alpha m_{3} \omega^{2}}$ \\
\hline
\end{tabular}

lopsidedness of the distribution, i.e. skewed to the right or to the left [18]. Using the Taylor polynomial approximation for cosine, we may relate $C(\omega)$ to the moments of $\hat{g}(v)$ :

$$
\begin{aligned}
C(\omega) & \approx \int_{0}^{\infty} \sum_{k=0}^{M} \frac{(-1)^{k}(\omega v)^{2 k}}{(2 k) !} \hat{g}(v) d v \\
& \approx \sum_{k=0}^{M} \frac{(-1)^{k} \omega^{2 k}}{(2 k) !} \int_{0}^{\infty} v^{2 k} \hat{g}(v) d v
\end{aligned}
$$

and similarly for $S(\omega)$. Using (3.1), it follows that

$$
\begin{aligned}
& C(\omega) \approx \sum_{k=0}^{M} \frac{(-1)^{k} \omega^{2 k}}{(2 k) !} m_{2 k} \\
& S(\omega) \approx \sum_{k=0}^{N} \frac{(-1)^{k} \omega^{2 k+1}}{(2 k+1) !} m_{2 k+1} .
\end{aligned}
$$

We define approximation $(M, N)$ as the approximation for $C(\omega)$ and $S(\omega)$ if we take the sum up to $M$ for the Taylor series for cosine and the sum up to $N$ for the Taylor series for sine. Note that these approximations should improve as $\omega$ approaches 0 or as $M$ and $N$ increase.

Thus, given knowledge of some moments of $\hat{g}(v)$, we may obtain an approximation for the curve(s) (2.9). The results for different cases are summarized in Table 1. In particular, we see that approximation $(0,0)$ is just a single point which corresponds to the characteristic equation having a double zero root. The other approximations are as follows

$$
\begin{array}{cl}
(1,0): \quad \tau=\frac{1}{\beta}, & \omega=\sqrt{\frac{2}{m_{2}}\left(1+\frac{\alpha}{\beta}\right)}, \\
(1,1): \quad \tau=\frac{1}{\left(1-\frac{m_{3}}{3 m_{2}}\right) \beta-\frac{m_{3}}{3 m_{2}} \alpha}, \quad \omega=\sqrt{\frac{2}{m_{2}}\left(1+\frac{\alpha}{\beta}\right)},
\end{array}
$$

where for $\alpha<0$ we require $\beta>-\alpha$ in order for $\omega$ to be defined. 
We note that approximation $(1,0)$ always underestimates the region of stability. For $\beta>|\alpha|$, this approximation recovers the results of Theorem 3. For $\beta<\alpha(\alpha>0)$ the curve enters the region of delay-independent stability and thus gives a worse estimate than Theorem 3 .

Approximation $(1,1)$ is a hyperbola with a vertical asymptote at $\beta=\nu \alpha /(1-\nu)$, where $\nu=m_{3} /\left(3 m_{2}\right)>0$ (since the moments are positive). The relationship between approximation $(1,1)$ and the results of Theorem 3 will depend on the value of $\nu$, and hence on the moments of the particular distribution. However, we can say that if $\nu<1$, then for large $\beta$, approximation $(1,1)$ always lies above approximation $(1,0)$. For $\beta<\alpha(\alpha>0)$ the curve given by approximation $(1,1)$ enters the region of distribution-independent stability if $\nu<1 / 2$.

Since it is known from Theorem 3 that, for $\alpha>0$, there is stability for $\beta<|\alpha|$ for any distribution, the best estimate for the stability region in this case is clearly to use the approximations above only for $\beta>|\alpha|$. This is what we do in the following sections where we apply the approximations to specific distributions.

\section{Verifying the approximation for the uniform distribution}

In this section we will apply the approximation procedure we derived in the previous section to the uniform distribution, thus determining approximations for the boundary of the region of stability. We will then compare these approximations with the true boundary derived from the characteristic equation.

The general uniform distribution with mean delay $\tau$ is given by

$$
g(u)=\left\{\begin{array}{cc}
\frac{1}{\rho \tau}, & \text { if } u \in\left[\tau\left(1-\frac{\rho}{2}\right), \tau\left(1+\frac{\rho}{2}\right)\right] \\
0, & \text { elsewhere }
\end{array}\right.
$$

where $0<\rho \leq 2$. Thus the normalized uniform distribution becomes

$$
\hat{g}(v)=\left\{\begin{array}{cc}
\frac{1}{\rho}, & \text { if } v \in\left[1-\frac{\rho}{2}, 1+\frac{\rho}{2}\right] \\
0, & \text { elsewhere. }
\end{array}\right.
$$

Hence the moments are given by

$$
\begin{aligned}
m_{k} & =\int_{0}^{\infty} v^{k} \hat{g}(v) d v=\frac{1}{\rho} \int_{1-\rho / 2}^{1+\rho / 2} v^{k} d v \\
& =\frac{1}{(k+1) \rho}\left[\left(1+\frac{\rho}{2}\right)^{k+1}-\left(1-\frac{\rho}{2}\right)^{k+1}\right] .
\end{aligned}
$$

The moments for some particular values of $\rho$ are given in Table 2 . 
Table 2: Moments of the uniform distribution.

\begin{tabular}{|c|c|c|c|c|}
\hline$\rho$ & $m_{0}$ & $m_{1}$ & $m_{2}$ & $m_{3}$ \\
\hline 2 & 1 & 1 & $4 / 3$ & 2 \\
\hline 1 & 1 & 1 & $13 / 12$ & $5 / 4$ \\
\hline $4 / 5$ & 1 & 1 & $79 / 75$ & $29 / 25$ \\
\hline
\end{tabular}

The corresponding approximations are

$$
\begin{aligned}
\rho=2 \quad(1,0): \quad \tau=\frac{1}{\beta}, \quad \omega=\sqrt{\frac{3}{2}\left(1+\frac{\alpha}{\beta}\right)} \\
(1,1): \quad \tau=\frac{2}{\beta-\alpha}, \quad \omega=\sqrt{\frac{3}{2}\left(1+\frac{\alpha}{\beta}\right)} \\
\rho=1 \quad(1,0): \quad \tau=\frac{1}{\beta}, \quad \omega=\sqrt{\frac{24}{13}\left(1+\frac{\alpha}{\beta}\right)} \\
(1,1): \quad \tau=\frac{13}{8 \beta-5 \alpha}, \quad \omega=\sqrt{\frac{24}{13}\left(1+\frac{\alpha}{\beta}\right)} \\
\quad(1,0): \quad \tau=\frac{1}{\beta}, \omega=\sqrt{\frac{150}{79}\left(1+\frac{\alpha}{\beta}\right)}, \\
(1,1): \quad \tau=\frac{79}{50 \beta-29 \alpha}, \quad \omega=\sqrt{\frac{150}{79}\left(1+\frac{\alpha}{\beta}\right)},
\end{aligned}
$$

where, for $\alpha<0$ we require $\beta>-\alpha$ for $\omega$ to exist. Approximation $(1,0)$ corresponds to the dotted line and approximation $(1,1)$ corresponds to the dashed black line in Figures $3-5$.

We now turn to the exact representation of the curves where the characteristic equation has a pair of pure imaginary roots. Since our scalar equation with uniform distributed delay is similar to the linearization of the delay equation used by Adimy et al. [2] to represent the dynamics of a pluripotent stem cell population, the computations that follow are similar to those found in [2]. For the uniform distribution (4.1) we have

$$
C(\omega)=\frac{1}{\rho} \int_{1-\rho / 2}^{1+\rho / 2} \cos (\omega v) d v=\frac{2 \cos (\omega) \sin (\rho \omega / 2)}{\rho \omega},
$$

and

$$
S(\omega)=\frac{1}{\rho} \int_{1-\rho / 2}^{1+\rho / 2} \sin (\omega v) d v=\frac{2 \sin (\omega) \sin (\rho \omega / 2)}{\rho \omega} .
$$

Substituting these into equations (2.9), we see that the curves are defined (parametrically in terms 
of $\omega)$ by

$$
\beta=-\frac{\alpha \rho \omega}{2 \cos (\omega) \sin (\rho \omega / 2)}, \quad \tau=-\frac{\omega \cos (\omega)}{\alpha \sin (\omega)} .
$$

To determine which curve(s) form part of the stability boundary we analyze the rate of change of the real part of the eigenvalues as one of the curves is crossed, using the formula (2.12) derived in the Section 2.

Now, from the expression for $\tau$ from (4.8) and equation (2.10), we have

$$
\frac{d \tau}{d \omega}=-\frac{\cos (\omega)}{\alpha \sin (\omega)}-\frac{\omega}{\alpha} \frac{\left(-\sin ^{2}(\omega)-\cos ^{2}(\omega)\right)}{\sin ^{2}(\omega)} .
$$

When $\alpha>0$, from (4.8) we need only consider $\omega$ values such that $\cos (\omega) / \sin (\omega)<0$, and thus

$$
\frac{d \tau}{d \omega}=\frac{1}{\alpha}\left(-\frac{\cos (\omega)}{\sin (\omega)}+\frac{\omega}{\sin ^{2}(\omega)}\right)>0
$$

When $\alpha<0$, we rewrite the derivative as

$$
\frac{d \tau}{d \omega}=\frac{1}{\alpha \sin ^{2}(\omega)}(\omega-\sin (\omega) \cos (\omega))
$$

But $\cos (\omega) / \sin (\omega)>0$ in this case, so

$$
\frac{\sin (\omega) \cos (\omega)}{\omega}=\left|\frac{\sin (\omega) \cos (\omega)}{\omega}\right|=\left|\frac{\sin (\omega)}{\omega}\right||\cos (\omega)|<1 .
$$

Thus $\omega-\sin (\omega) \cos (\omega)>0$ and $\frac{d \tau}{d \omega}<0$. We therefore determine that $\alpha \frac{d \tau}{d \omega}>0$, for any value of $\alpha$. Using this and the fact that we only consider $\beta>0$, we conclude from (2.12) that

$$
\left.\frac{d \operatorname{Re}(\lambda)}{d \beta}\right|_{\lambda=i \omega}>0
$$

In other words, the real part of $\lambda$ increases (decreases) as $\beta$ increases (decreases), as the curves where $\lambda=i \omega$ are crossed. It follows from this computation that the boundary of the stability region is formed by the curve closest to the $\tau$ axis.

For $\rho=2$, the parametric equations in (4.8) become

$$
\beta=-\frac{\alpha \omega}{\cos (\omega) \sin (\omega)}, \quad \tau=-\frac{\omega \cos (\omega)}{\alpha \sin (\omega)} .
$$

Due to the singularities at $\omega=k \pi$ and $\omega=(2 k+1) \pi / 2$ for $k=0,1, \ldots$, these equations define multiple curves in the $\beta, \tau$ plane, which lie either in the first or third quadrant. Since we are interested in $\tau>0$, the only curves of interest are those in the first quadrant.

For $\alpha<0$, the curve forming the boundary of the stability region is defined by equation (4.9) with $\omega \in\left[0, \frac{\pi}{2}\right]$ and for $\alpha>0$ with $\omega \in\left[\frac{\pi}{2}, \pi\right]$. Some simple properties of this curve are as follows. 


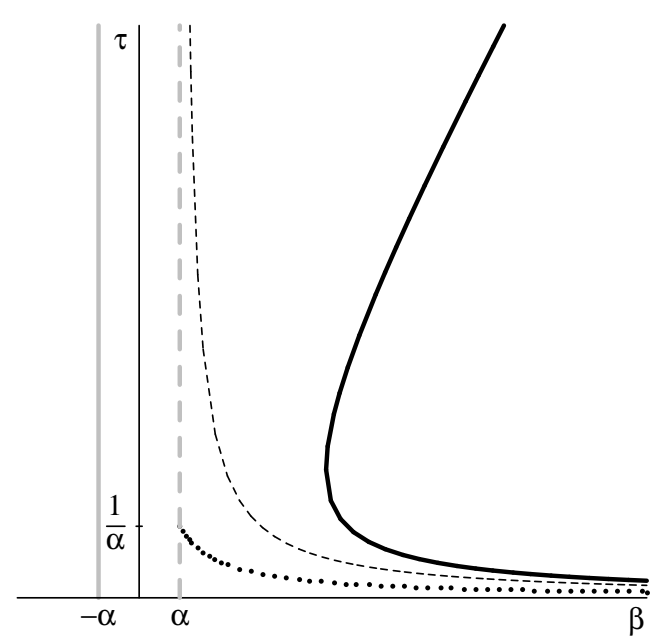

(a) $\alpha>0$

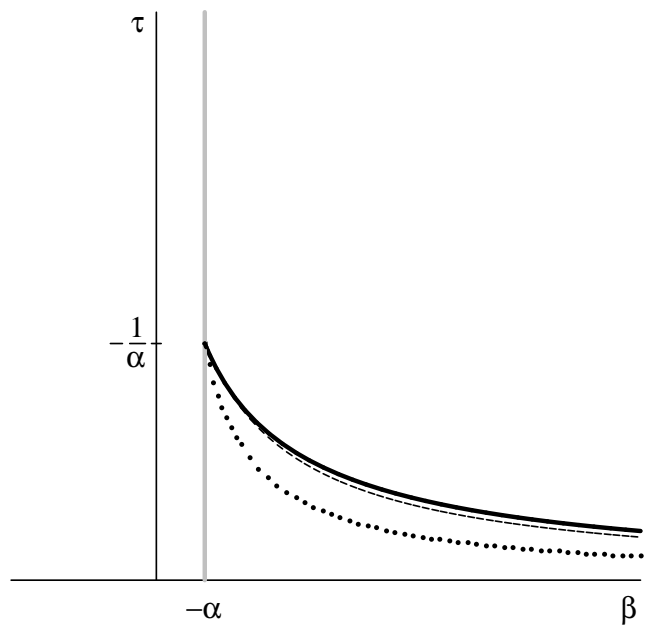

(b) $\alpha<0$

Figure 3: Stability region for the uniform distribution with $\rho=2$. The region of distribution independent stability lies between the solid and dashed gray lines when $\alpha>0$. The true region of stability lies between the solid gray line and the solid black curve which is defined by equations (4.9). The dotted curve, defined by equation (4.2), shows the first approximation to the solid black curve. The dashed black curve, defined by equation (4.3), shows the second approximation.

For $\alpha<0$, the curve approaches the point $(\beta, \tau)=(-\alpha,-1 / \alpha)$ as $\omega \rightarrow 0$. For any $\alpha$, the curve has a horizontal asymptote, $\tau=0$, which is approached as $\omega \rightarrow \frac{\pi}{2}$. For $\alpha>0$ it has an oblique asymptote, $\tau=\beta / \alpha^{2}$, which is approached as $\omega \rightarrow \pi$. This curve corresponds to the solid black lines in Figure 3.

For $\rho=1$, the parametric equations in (4.8) are given by

$$
\beta=-\frac{\alpha \omega / 2}{\cos (\omega) \sin (\omega / 2)}, \quad \tau=-\frac{\omega \cos (\omega)}{\alpha \sin (\omega)} .
$$

These equations define multiple curves in the $\beta, \tau$ plane with singularities at $\omega=k \pi$ and $\omega=$ $(2 k+1) \pi / 2$ for $k=0,1, \ldots$. For $\alpha<0$ the closest curve to the $\tau$ axis is defined by equation (4.10) with $\omega \in\left[0, \frac{\pi}{2}\right]$ and for $\alpha>0$ with $\omega \in\left[\frac{\pi}{2}, \pi\right]$. For $\alpha<0$, the curve approaches the point $(\beta, \tau)=(-\alpha,-1 / \alpha)$ as $\omega \rightarrow 0$. For any $\alpha$, the curves in (4.10) have a horizontal asymptote, $\tau=0$, which is approached as $\omega \rightarrow(2 k+1) \pi / 2$, vertical asymptotes as $\omega \rightarrow(2 k+1) \pi$, and an oblique asymptote as $\omega \rightarrow 2 k \pi$. These curves correspond to the solid black lines in Figure 4.

For $\rho=4 / 5$, the parametric equations in (4.8) become

$$
\beta=-\frac{2 \alpha \omega / 5}{\cos (\omega) \sin (2 \omega / 5)}, \quad \tau=-\frac{\omega \cos (\omega)}{\alpha \sin (\omega)} .
$$

These equations define multiple curves in the $\beta, \tau$ plane with singularities at $\omega=k \pi$ and $\omega=$ $(2 k+1) \pi / 2$ for $k=0,1, \ldots$ For $\alpha<0$ this curve closest to the $\tau$ axis is defined by equation (4.11) 


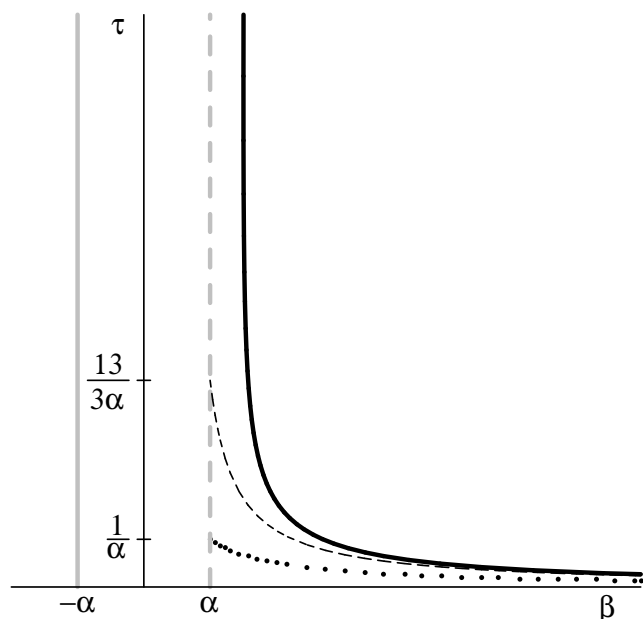

(a) $\alpha>0$

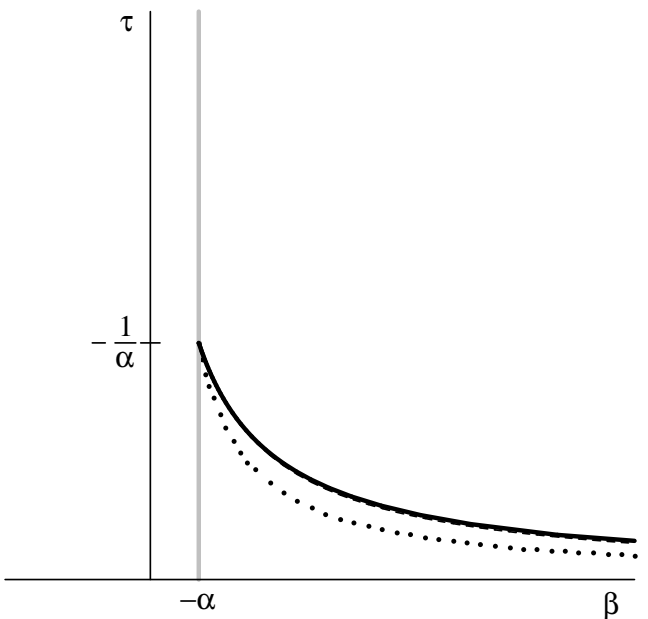

(b) $\alpha<0$

Figure 4: Stability region for the uniform distribution with $\rho=1$. The region of distribution independent stability lies between the solid and dashed gray lines when $\alpha>0$. The true region of stability lies between the solid gray line and the solid black curve which is defined by equations (4.10). The dotted curve, defined by equation (4.4), shows the first approximation to the solid black curve. The dashed black curve, defined by equation (4.5), shows the second approximation.

with $\omega \in\left[0, \frac{\pi}{2}\right]$ and for $\alpha>0$ with $\omega \in\left[\frac{\pi}{2}, \pi\right]$. For $\alpha<0$, the curve approaches the point $(\beta, \tau)=(-\alpha,-1 / \alpha)$ as $\omega \rightarrow 0$. For any $\alpha$, the curves in (4.11) have a horizontal asymptote, $\tau=0$, which is approached as $\omega \rightarrow(2 k+1) \pi / 2$ and vertical asymptotes as $\omega \rightarrow(2 k+1) \pi$. These curves correspond to the solid black lines in Figure 5.

Comparing the approximations with the true boundary of the stability region in Figures $3-5$ we can make several conclusions. The approximations improve as the number of moments used increases. This corresponds to increasing $N$ and $M$ in the approximations for sine and cosine and is as expected. The approximations are better for large $\beta$. In all cases, larger $\beta$ corresponds to smaller $\omega$ so this is also as should be expected. Note also that the approximate stability regions are always conservative, i.e. they underestimate the region of of stability.

\section{Verifying the approximation for the gamma distribution}

In this section we will apply the approximation procedure we derived in Section 3 to the gamma distribution, thus determining approximations for the boundary of the region of stability. We will then compare these approximations with the true boundary derived from the characteristic equation. 


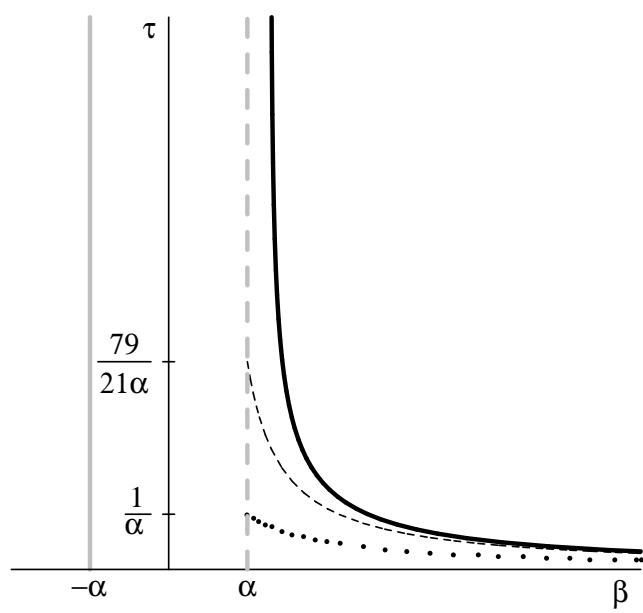

(a) $\alpha>0$

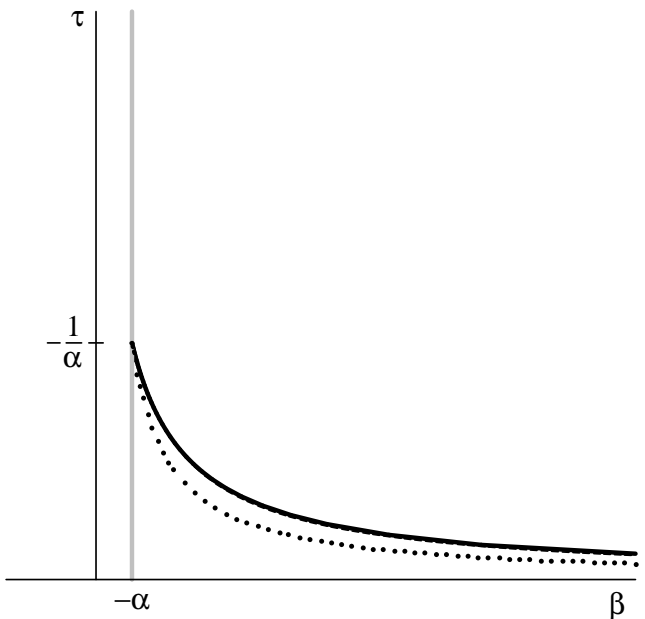

(b) $\alpha<0$

Figure 5: Stability region for the uniform distribution with $\rho=0.8$. The region of distribution independent stability lies between the solid and dashed gray lines when $\alpha>0$. The true region of stability lies between the solid gray line and the solid black curve which is defined by equations (4.11). The dotted curve, defined by equation (4.6), shows the first approximation to the solid black curve. The dashed black curve, defined by equation (4.7), shows the second approximation.

Recall that the gamma distribution is given by

$$
g(u)=\frac{u^{p-1} a^{p} e^{-a u}}{(p-1) !}
$$

with mean delay $\tau=p / a$. Thus the scaled distribution is given by

$$
\begin{aligned}
\hat{g}(v) & =\tau g(\tau v) \\
& =\frac{(\tau a)^{p} v^{p-1} e^{-a \tau v}}{(p-1) !} \\
& =\frac{p^{p} v^{p-1} e^{-p v}}{(p-1) !},
\end{aligned}
$$

and the $k^{\text {th }}$ moment is given by

$$
\begin{aligned}
m_{k} & =\int_{0}^{\infty} v^{k} \hat{g}(v) d v \\
& =\frac{p^{p}}{(p-1) !} \int_{0}^{\infty} v^{k+p-1} e^{-p v} d v .
\end{aligned}
$$

To find a general expression for $m_{k}$ we will use the following result, which is easily derived by mathematical induction,

$$
\int_{0}^{\infty} v^{n} e^{-p v} d v=\frac{n !}{p^{n+1}}
$$


Using this in (5.2) we arrive at an expression for the $k^{\text {th }}$ moment of the gamma distribution:

$$
m_{k}=p^{-k} \frac{(k+p-1) !}{(p-1) !}
$$

Table 3 gives values of these moments for various values of $p$. The corresponding approximations

Table 3: Moments of the gamma distribution.

\begin{tabular}{|c|c|c|c|c|}
\hline$p$ & $m_{0}$ & $m_{1}$ & $m_{2}$ & $m_{3}$ \\
\hline 1 & 1 & 1 & 2 & 6 \\
\hline 2 & 1 & 1 & $3 / 2$ & 3 \\
\hline 3 & 1 & 1 & $4 / 3$ & $20 / 9$ \\
\hline
\end{tabular}

are

$$
\begin{aligned}
p=1 \quad(1,0): \quad \tau & =\frac{1}{\beta}, \quad \omega=\sqrt{1+\frac{\alpha}{\beta}} \\
(1,1): \quad \tau & =-\frac{1}{\alpha}, \quad \omega=\sqrt{1+\frac{\alpha}{\beta}} \\
p=2 \quad(1,0): \quad \tau & =\frac{1}{\beta}, \quad \omega=\sqrt{\frac{4}{3}\left(1+\frac{\alpha}{\beta}\right)} \\
(1,1): \quad \tau & =\frac{3}{\beta-2 \alpha}, \quad \omega=\sqrt{\frac{4}{3}\left(1+\frac{\alpha}{\beta}\right)}, \\
p=3 \quad(1,0): \quad \tau & =\frac{1}{\beta}, \omega=\sqrt{\frac{3}{2}\left(1+\frac{\alpha}{\beta}\right)} \\
(1,1): \quad \tau & =\frac{9}{4 \beta-5 \alpha}, \quad \omega=\sqrt{\frac{3}{2}\left(1+\frac{\alpha}{\beta}\right)},
\end{aligned}
$$

where, for $\alpha<0$ we impose that $\beta>-\alpha$ in order for $\omega$ to be defined. Approximations $(1,0)$ are shown with dotted lines and approximations $(1,1)$ are shown with dashed black lines in Figures 6 8 .

We now turn to the exact representation of the curves where the characteristic equation has a pair of pure imaginary roots. For the gamma distribution (5.1) we have

$$
\begin{aligned}
C(\omega) & =\operatorname{Re}\left(\int_{0}^{\infty} \hat{g}(v) e^{-i \omega v} d v\right) \\
& =\operatorname{Re}\left(\frac{p^{p}}{(p-1) !} \int_{0}^{\infty} v^{p-1} e^{-(p+i \omega) v} d v\right) \\
& =\left(1+\frac{\omega^{2}}{p^{2}}\right)^{-p} \operatorname{Re}\left(1-\frac{i \omega}{p}\right)^{p}
\end{aligned}
$$


Table 4: Expressions for $C(\omega)$ and $S(\omega)$ for the gamma distribution with $p=1,2,3$.

\begin{tabular}{|c|c|c|}
\hline$p$ & $C(\omega)$ & $S(\omega)$ \\
\hline 1 & $\frac{1}{1+\omega^{2}}$ & $\frac{\omega}{1+\omega^{2}}$ \\
\hline 2 & $\frac{1-\omega^{2} / 4}{\left(1+\omega^{2} / 4\right)^{2}}$ & $\frac{\omega}{\left(1+\omega^{2} / 4\right)^{2}}$ \\
\hline 3 & $\frac{1-\omega^{2} / 3}{\left(1+\omega^{2} / 9\right)^{3}}$ & $\frac{\omega\left(1-\omega^{2} / 27\right)}{\left(1+\omega^{2} / 9\right)^{3}}$ \\
\hline
\end{tabular}

using equation (5.3). Similarly, $S(\omega)=-\left(1+\frac{\omega^{2}}{p^{2}}\right)^{-p} \operatorname{Im}\left(1-\frac{i \omega}{p}\right)^{p}$. The expressions for $C(\omega)$ and $S(\omega)$ for various values of $p$ are given in Table 4. Using these expressions we arrive at the parametric equations for $\tau$ and $\beta$.

For $p=1$ we have

$$
\tau=-\frac{1}{\alpha}, \omega^{2}=-\left(1+\frac{\beta}{\alpha}\right) .
$$

Note that this implies that there are no points where the characteristic equation has pure imaginary roots in the region $\tau \geq 0$ if $\alpha>0$. If $\alpha<0, \omega$ is only defined for $\beta>-\alpha$, so the stability boundary can only include this part of the curve. A simple calculation shows that

$$
\begin{aligned}
\left.\frac{d \operatorname{Re}(\lambda)}{d \tau}\right|_{\lambda=i \omega} & =-\left.\operatorname{Re}\left(\frac{\partial \Delta}{\partial \tau} / \frac{\partial \Delta}{\partial \lambda}\right)\right|_{\lambda=i \omega} \\
& =-\operatorname{Re}\left(\frac{(\alpha+\beta)+i(\alpha \omega)}{i(2 \omega)}\right) \\
& =-\frac{\alpha}{2}>0,
\end{aligned}
$$

since $\alpha<0$. We obtain the region of stability for $p=1$ as seen in Figure 6. For $\alpha>0$ the equilibrium point is locally asymptotically stable when $\beta>-\alpha$, and for $\alpha<0$ it is locally asymptotically stable when $\beta>-\alpha$ and $\tau<-1 / \alpha$. Recall that the approximations are given by equations (5.4) and (5.5). Thus the estimate for the region of stability for $p=1$ given by the second approximation is identical to the true region of stability obtained analytically using full knowledge of the distribution.

For $p=2$ the curve where the characteristic equation has a pair of pure imaginary roots is defined by

$$
\beta=\frac{(\alpha \tau+2)^{2}}{\tau}, \omega^{2}=4(\alpha \tau+1) .
$$

If $\alpha>0, \omega$ is defined for all $\tau$ and the curve is a parabola with minimum at $\beta=8 \alpha$ and $\tau=2 / \alpha$. If $\alpha<0, \omega$ is defined for $\tau<-1 / \alpha$ and the curve is a parabola with minimum at $\beta=0$ and 


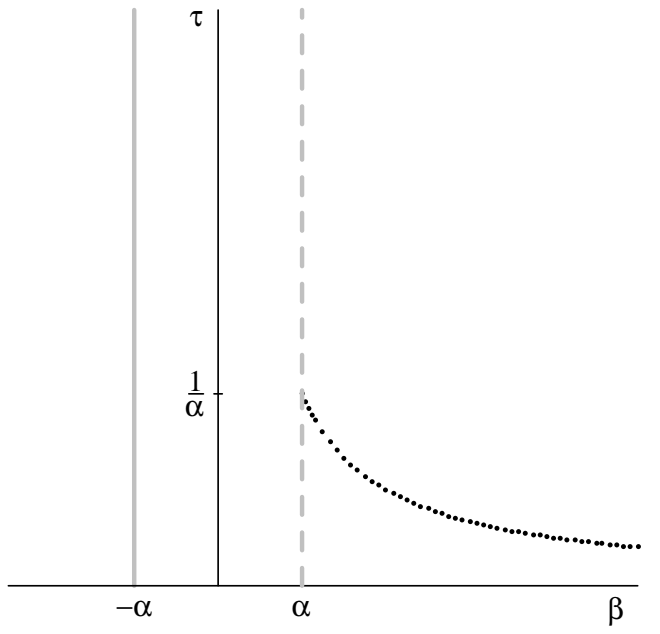

(a) $\alpha>0$

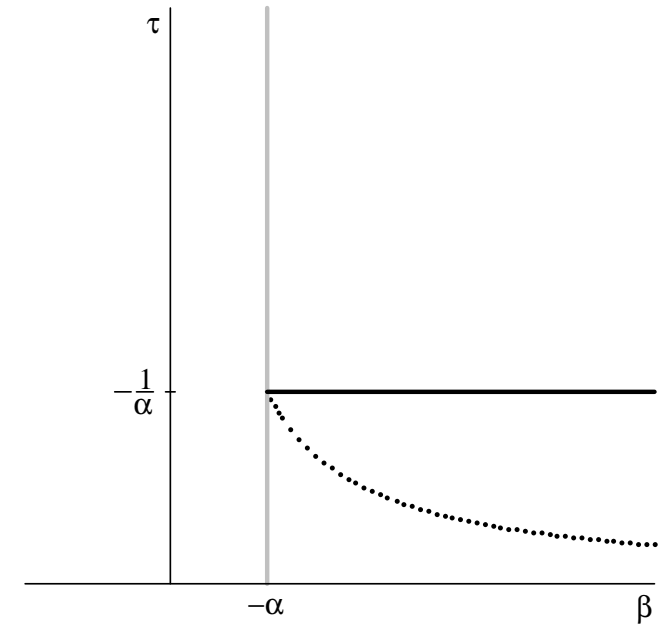

(b) $\alpha<0$

Figure 6: Stability region for the gamma distribution with $p=1$. When $\alpha>0$, the region of distribution independent stability lies between the solid and dashed gray lines. For $\alpha>0$, the true region of stability lies to the left the the solid gray line, and for $\alpha<0$, it lies between the solid gray line and the solid black curve defined by equations (5.10). The dotted curve, defined by (5.4), shows the first approximation to the boundary of the stability region. In both cases, the second approximation, defined by equation (5.5), recovers the full stability region.

$\tau=-2 / \alpha$. To obtain the rate of change of the real part of $\lambda$ with respect to $\beta$ we first compute

$$
C^{\prime}(\omega)=\frac{8 \omega\left(\omega^{2}-12\right)}{\left(\omega^{2}+4\right)^{3}} \text { and } S^{\prime}(\omega)=\frac{16\left(4-3 \omega^{2}\right)}{\left(\omega^{2}+4\right)^{3}} .
$$

Substituting these and the values of $C(\omega)$ and $S(\omega)$ from Table 4 into (2.11) we obtain

$$
\left.\frac{d \operatorname{Re}(\lambda)}{d \beta}\right|_{\lambda=i \omega}=-\frac{\tau}{H^{2}(\omega)}\left(-\frac{8 \omega^{2}}{\left(\omega^{2}-4\right)^{2}}\right)>0 .
$$

We determine the region of stability for $p=2$ as seen in Figure 7. For $\alpha>0$ the equilibrium point is locally asymptotically stable when

$$
\beta>-\alpha \text { and } \tau<\frac{\beta-4 \alpha-\sqrt{\beta^{2}-8 \alpha \beta}}{2 \alpha^{2}} \text { or } \tau>\frac{\beta-4 \alpha+\sqrt{\beta^{2}-8 \alpha \beta}}{2 \alpha^{2}} .
$$

For $\alpha<0$ it is locally asymptotically stable when

$$
\beta>-\alpha \text { and } \tau<\frac{\beta-4 \alpha-\sqrt{\beta^{2}-8 \alpha \beta}}{2 \alpha^{2}} .
$$

We note that for $\alpha>0$ the stability is recovered as $\tau$ is sufficiently increased, but for $\alpha<0$ stability is lost as soon as $\tau$ crosses the lower branch of the parabola. 


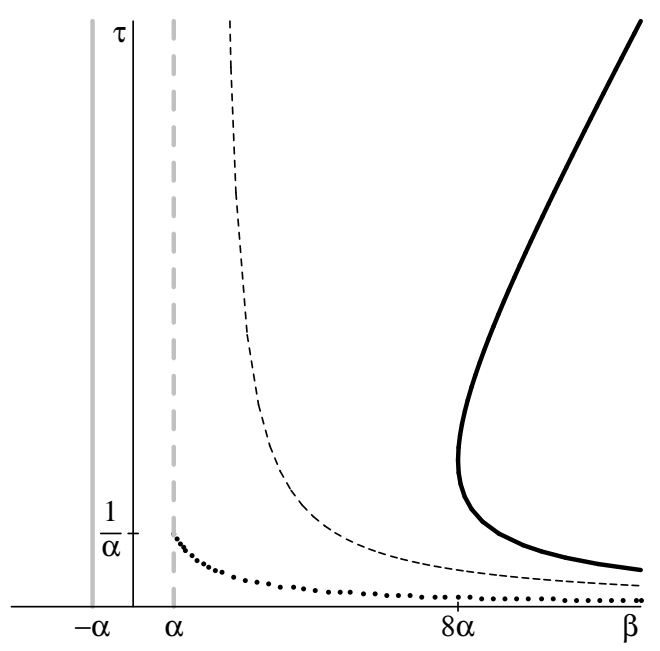

(a) $\alpha>0$

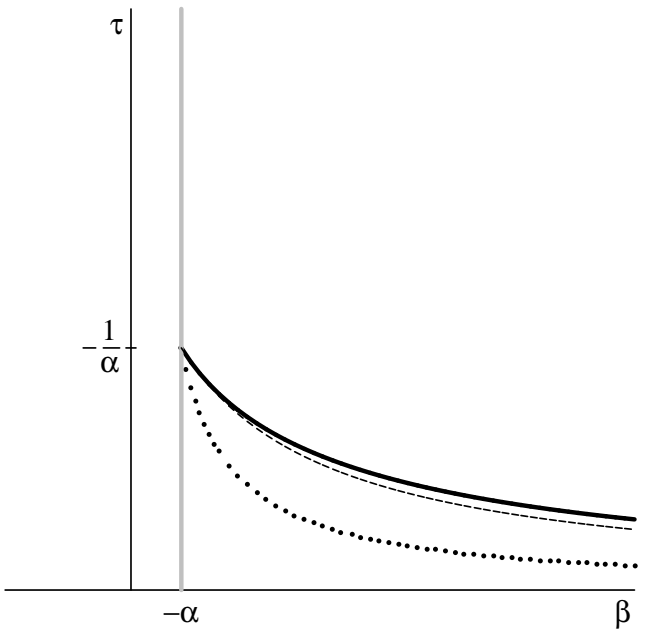

(b) $\alpha<0$

Figure 7: Stability region for the gamma distribution with $p=2$. The region of distribution independent stability lies between the solid and dashed gray lines when $\alpha>0$. The true region of stability lies between the solid gray line and the solid black curve which is defined by equations (5.11). The dotted curve, defined by equation (5.6), shows the first approximation to the solid black curve. The dashed black curve, defined by equation (5.7), shows the second approximation.

Next we will consider how well the approximations curves estimate this region of stability. Recall that the approximation curves are given by (5.6) and (5.7). These are shown in Figure 7 by the dotted curve representing approximation $(1,0)$ and the dashed black curve representing approximation $(1,1)$. For $\alpha>0$, both approximations estimate the region of stability well for large $\beta$. When $\alpha<0$, the two approximation curves give good estimates for any $\beta>-\alpha$. We note that the second approximation gives a better estimate than the first approximation, as expected, and that both curves give smaller regions of stability than the exact curves.

Finally, for $p=3$ we have

$$
\beta=\frac{8(\alpha \tau+3)^{3}}{\tau(\alpha \tau+9)^{2}}, \omega^{2}=\frac{27(\alpha \tau+1)}{\alpha \tau+9} .
$$

For $\alpha>0, \omega$ is defined for all $\tau \geq 0$ and the curve lies in the first quadrant with a horizontal asymptote at $\tau=0$ and a vertical asymptote at $\beta=8 \alpha$. For $\alpha<0, \omega$ is defined for $\tau \leq-1 / \alpha$ which corresponds to $\beta \geq-\alpha$, or $\tau>-9 / \alpha$ which corresponds to $\beta<8 \alpha$. However, as noted above, we only consider curves where $\beta$ is positive, and thus only the portion of the curve with $\tau<-1 / \alpha$ will form part of the stability boundary. This curve has a horizontal asymptote at $\tau=0$. Further we have that

$$
C^{\prime}(\omega)=\frac{927 \omega\left(\omega^{2}-9\right)}{\left(\omega^{2}+9\right)^{4}} \text { and } S^{\prime}(\omega)=\frac{81\left(\omega^{4}-54 \omega^{2}+81\right)}{\left(\omega^{2}+9\right)^{4}} .
$$


Substituting these and the values of $C(\omega)$ and $S(\omega)$ from Table 4 into (2.11) and, using (5.12), we obtain

$$
\begin{aligned}
\left.\frac{d \operatorname{Re}(\lambda)}{d \beta}\right|_{\lambda=i \omega} & =-\frac{\tau}{H^{2}(\omega)}\left(\frac{11664 \omega^{2}}{\left(\omega^{2}+9\right)^{3}\left(\omega^{2}-27\right)}\right) \\
& =\frac{\tau}{H^{2}(\omega)}\left(\frac{54 \omega^{2}(\alpha \tau+9)}{\left(\omega^{2}+9\right)^{3}}\right) .
\end{aligned}
$$

But $\alpha \tau+9>0$ for $\alpha>0$ and also for $\alpha<0$ since we are only considering $\tau \leq-1 / \alpha$. Therefore

$$
\left.\frac{d \operatorname{Re}(\lambda)}{d \beta}\right|_{\lambda=i \omega}>0 .
$$

The region of stability for $p=3$ can be as seen in Figure 8. For $\alpha>0$ the equilibrium point is locally asymptotically stable when

$$
\beta>-\alpha \text { and } \beta<\frac{8(\alpha \tau+3)^{3}}{\tau(\alpha \tau+9)^{2}} .
$$

For $\alpha<0$ the equilibrium point is locally asymptotically stable when

$$
\beta>-\alpha \text { and } \beta<\frac{8(\alpha \tau+3)^{3}}{\tau(\alpha \tau+9)^{2}} \text { with } \tau<-1 / \alpha,
$$

i.e., $\tau$ is underneath the solid line as seen in Figure 8. We note that for $\alpha>0$, if $\beta<8 \alpha$ then stability is always recovered when $\tau$ is sufficiently large, but for $\alpha<0$ stability is lost as soon as $\tau$ crosses the solid curve.

We next examine the approximation curves given by equations (5.8) and (5.9). These are shown in Figure 8 by the dotted and dashed black curves. For $\alpha>0$, both approximations give a good estimate of the region of stability for large $\beta$. When $\alpha<0$, the two approximation curves estimate the region of stability well for any $\beta>-\alpha$. We note that, again, the second approximation gives a better estimate than the first approximation, and that both curves give smaller regions of stability than the exact curves.

\section{Conclusions}

We have studied the linear stability analysis of a scalar differential equation with one distributed delay. While our results are specific to this differential equation, our methods are general and could be applied to equations of higher order and/or with more than one distributed delay. These methods and results are summarized below.

We have shown how to obtain the distribution independent region of stability of an equilibrium point. This region is similar to the delay independent region of stability for equations with discrete delays. In fact, for the equation we studied, the distribution independent region of stability is the 


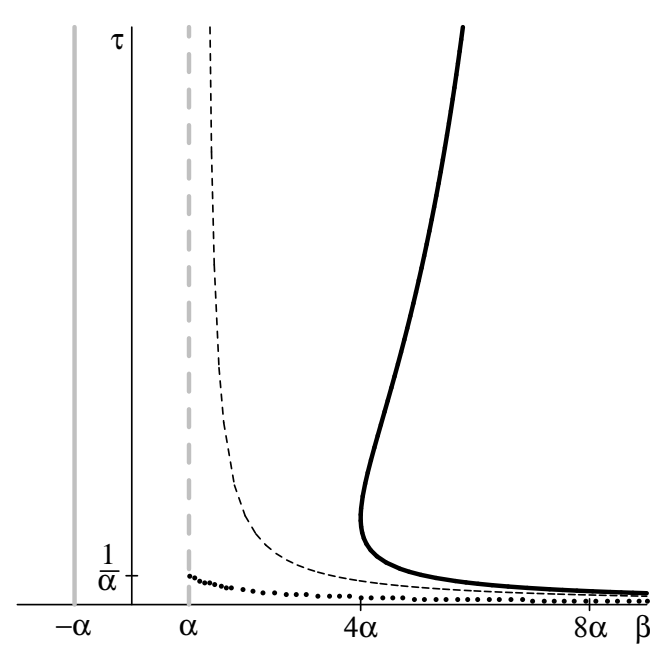

(a) $\alpha>0$

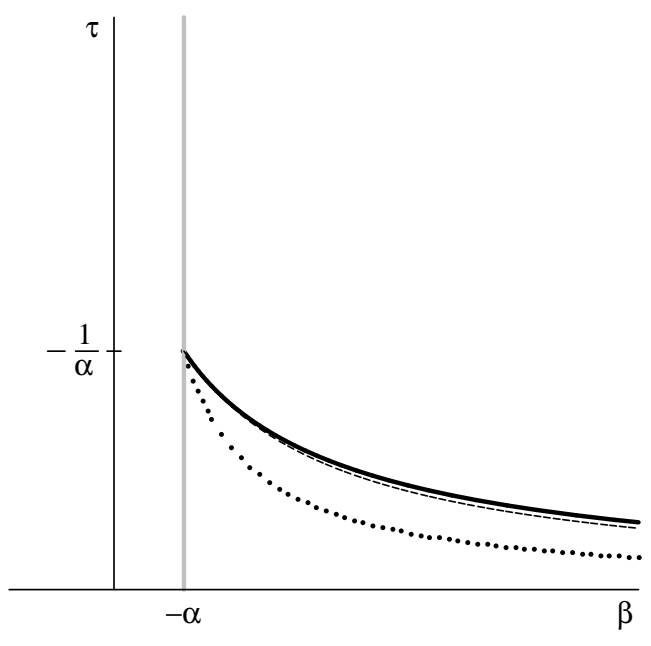

(b) $\alpha<0$

Figure 8: Stability region for the gamma distribution with $p=3$. The region of distribution independent stability lies between the solid and dashed gray lines when $\alpha>0$. The true region of stability lies between the solid gray line and the solid black curve which is defined by equations (5.12). The dotted curve, defined by equation (5.8), shows the first approximation to the solid black curve. The dashed black curve, defined by equation (5.9), shows the second approximation.

same as the delay independent region if the distributed delay is replaced by a discrete delay equal to the mean of the distribution.

We have shown how to reformulate the distribution so that the mean delay occurs as a natural parameter in the distribution. This allows us to determine a region of stability which depends on the mean delay, but is independent of other properties of the distribution.

Both the distribution independent region of stability and the mean delay dependent region are conservative estimates of the full region of stability of the equilibrium point. Thus we formulated an approach to approximate the boundary of the full region of stability based on a finite number of moments of the distribution. By comparing our approximation with the true stability region boundary calculated for the uniform and gamma distributions, we show that the approximation improves as more moments are included, as might be expected. The accuracy of a given approximation is not uniform in the parameters of the system, however, we can predict where it should be better and worse.

We feel that the distribution independent and approximation approaches may be valuable for studying models of real applications. In such situations, the exact distribution is generally unknown, but it may be reasonable to obtain the mean, variance and possibly other moments of the distribution. 


\section{Acknowledgements}

We thank the anonymous reviewers for their careful reading of our manuscript and for bringing several relevant references to our attention. SAC acknowledges support of NSERC. RJ acknowledges support of OGSST.

\section{References}

[1] M. Adimy, F. Crauste, S. Ruan. A mathematical study of the hematopoiesis process with applications to chronic myelogenous leukemia. SIAM J. Appl. Math., 65 (2005), 1328-1352.

[2] M. Adimy, F. Crauste, S. Ruan. Stability and Hopf bifurcation in a mathematical model of pluripotent stem cell dynamics. Nonl. Anal.: Real World Appl., 6 (2005), 651-670.

[3] J. Arino, P. van den Driessche. Time delays in epidemic models: modeling and numerical considerations, in Delay differential equations and applications, chapter 13, 539-558. Springer, Dordrecht, 2006.

[4] F.M. Atay. Distributed delays facilitate amplitude death of coupled oscillators. Phys. Rev. Lett., 91 (2003), 094101.

[5] F.M. Atay. Oscillator death in coupled functional differential equations near Hopf bifurcation. J. Diff. Eqs., 221 (2006), 190-209.

[6] F.M. Atay. Delayed feedback control near Hopf bifurcation. DCDS, 1 (2008), 197-205.

[7] S. Bernard, J. Bélair, M.C. Mackey. Sufficient conditions for stability of linear differential equations with distributed delay. DCDS, 1B (2001), 233-256.

[8] F. Brauer, C. Castillo-Chávez. Mathematical models in population biology and epidemiology. Springer, New York, 2001.

[9] S.A. Campbell, I. Ncube. Some effects of gamma distribution on the dynamics of a scalar delay differential equation. Preprint, (2009).

[10] Y. Chen. Global stability of neural networks with distributed delays. Neur. Net., 15 (2002), 867-871.

[11] Y. Chen. Global stability of delayed Cohen-Grossberg neural networks. IEEE Trans. Circuits Syst.-I, 53 (2006), 351-357.

[12] R.V. Churchill, J.W. Brown. Complex variables and applications. McGraw-Hill, New York, 1984.

[13] K.L. Cooke, Z. Grossman. Discrete delay, distributed delay and stability switches. J. Math. Anal. Appl., 86 (1982), 592-627. 
[14] J.M. Cushing. Integrodifferential equations and delay models in population dynamics, Vol. 20 of Lecture Notes in Biomathematics. Springer-Verlag, Berlin, New York, 1977.

[15] T. Faria, J.J. Oliveira. Local and global stability for Lotka-Volterra systems with distributed delays and instantaneous negative feedbacks. J. Diff. Eqs., 244 (2008), 1049-1079.

[16] K. Gopalsamy. Stability and oscillations in delay differential equations of population dynamics. Kluwer, Dordrecht, 1992.

[17] K. Gopalsamy and X.-Z. He. Stability in asymmetric Hopfield nets with transmission delays. Physica D, 76 (1994), 344-358.

[18] R.V. Hogg and A.T. Craig. Introduction to mathematical statistics. Prentice Hall, United States, 1995.

[19] G.E. Hutchinson. Circular cause systems in ecology. Ann. N.Y. Acad. Sci., 50 (1948), 221246.

[20] V.K. Jirsa, M. Ding. Will a large complex system with delays be stable?. Phys. Rev. Lett., 93 (2004), 070602.

[21] K. Koch. Biophysics of computation: information processing in single neurons. Oxford University Press, New York, 1999.

[22] Y. Kuang. Delay differential equations: with applications in population dynamics, Vol. 191 of Mathematics in Science and Engineering. Academic Press, New York, 1993.

[23] X. Liao, K.-W. Wong, Z. Wu. Bifurcation analysis on a two-neuron system with distributed delays. Physica D, 149 (2001), 123-141.

[24] N. MacDonald. Time lags in biological models, Vol. 27 of Lecture Notes in Biomathematics. Springer-Verlag, Berlin; New York, 1978.

[25] N. MacDonald. Biological delay systems: linear stability theory. Cambridge University Press, Cambridge, 1989.

[26] M.C. Mackey, U. an der Heiden. The dynamics of recurrent inhibition. J. Math. Biol., 19 (1984), 211-225.

[27] J.M. Milton. Dynamics of small neural populations, Vol. 7 of CRM monograph series. American Mathematical Society, Providence, 1996.

[28] S. Ruan. Delay differential equations for single species dynamics, in Delay differential equations and applications, chapter 11, 477-515. Springer, Dordrecht, 2006.

[29] S. Ruan, R.S. Filfil. Dynamics of a two-neuron system with discrete and distributed delays. Physica D, 191 (2004), 323-342. 
[30] A. Thiel, H. Schwegler, C.W. Eurich. Complex dynamics is abolished in delayed recurrent systems with distributed feedback times. Complexity, 8 (2003), 102-108.

[31] G.S.K. Wolkowicz, H. Xia, S. Ruan. Competition in the chemostat: A distributed delay model and its global asymptotic behaviour. SIAM J. Appl. Math., 57 (1997), 1281-1310.

[32] G.S.K. Wolkowicz, H. Xia, J. Wu. Global dynamics of a chemostat competition model with distributed delay. J. Math. Biol., 38 (1999), 285-316.

[33] P. Yan. Separate roles of the latent and infectious periods in shaping the relation between the basic reproduction number and the intrinsic growth rate of infectious disease outbreaks. $\mathrm{J}$. Theoret. Biol., 251 (2008), 238-252. 